\title{
Correlation between magnetic and transport properties in nanocrystalline Fe thin films: A grain-boundary magnetic disorder effect
}

\author{
Z. Sefrioui, ${ }^{1, *}$ J. L. Menéndez, ${ }^{1}$ E. Navarro, ${ }^{1,2}$ A. Cebollada, ${ }^{1}$ F. Briones, ${ }^{1}$ P. Crespo, ${ }^{3}$ and A. Hernando ${ }^{3}$ \\ ${ }^{1}$ Instituto de Microelectrónica de Madrid-IMM (CNM-CSIC), Isaac Newton 8 (PTM) Tres Cantos, Madrid 28760, Spain \\ ${ }^{2}$ Dept. Física Aplicada, Facultad de Veterinaria, Universidad Complutense, 28040 Madrid, Spain \\ ${ }^{3}$ Instituto de Magnetismo Aplicado, U.C.M., PO Box 155, 28230 Las Rozas, Madrid, Spain
}

(Received 10 July 2001; published 26 November 2001)

\begin{abstract}
We report on transport and magnetic measurements of islanded Fe(110) thin films. The electrical resistivity exhibits an anomalous increase at low temperatures, which disappears under the action of a magnetic field. Since such an anomaly completely disappears under the action of a magnetic field, it is inferred that it originates from spin-dependent scattering. We interpret the strong changes in the spin-dependent scattering in our films to be due to a low-temperature spin freezing of the island boundary magnetic regions that impedes ferromagnetic exchange between islands. A consequence of this magnetic behavior is the random arrangement of the individual magnetization, determined by the magnetocrystalline anisotropy of each island, resulting in an increase of the resistivity below the freezing temperature.
\end{abstract}

DOI: 10.1103/PhysRevB.64.224431

PACS number(s): 75.70.-i, 75.50.Bb

Phenomena in nanostructured magnetic systems have attracted much attention in recent years, especially those in which spin-dependent scattering plays an important role. For example, the influence of magnetic disorder in spindependent scattering shows its clear manifestation in the well-known giant magnetoresistance effect. ${ }^{1-4}$ This phenomenon is due to the change in resistivity of systems in which the magnetization directions of individual magnetic entities can be aligned on misaligned with respect to each other upon application of a magnetic field. These magnetic entities can indeed be of diverse natures: layers separated by nanometerthick nonferromagnetic spacers in a multilayer or a spin valve, ${ }^{1,5,6}$ grains embedded in a matrix to constitute a granular system, ${ }^{7-11}$ or even magnetic domains separated by domain walls in a continuous ferromagnetic film. ${ }^{12}$ In all these cases, the observed magnetoresistance is due to a transition from a magnetically disordered state (antiparallel alignment in a multilayer or spin valve, random orientation in a granular system or multidomain state in a ferromagnetic thin film) to an ordered one (a parallel configuration or single-domain state). In all these cases, a key parameter is the spin diffusion length, which determines an upper limit for the extension of the magnetic disorder to give rise to magnetoresistive effects. It is because of the limited value of this magnitude, in the range of a few nanometers, that a manifestation of these phenomena is restricted to systems in which at least one of the dimensions is structured in the nanometer range. On the other hand, nanostructured materials with characteristic structural coherence lengths (such as grain size) in the range of a few to a few tens of nanometers are also very interesting, because the scale of the microstructure is comparable to the ferromagnetic exchange length as well as because the large relative fraction of atoms at the interfaces. As a consequence, dramatic changes in the magnetic behavior are driven by small variations in both grain size and magnetic correlation across grain boundaries. ${ }^{13}$ As recently reported, ${ }^{14}$ nanocrystalline Fe obtained by ball milling exhibits a lowtemperature magnetic hardening. Magnetic decoupling due to a spin freezing of the grain boundaries was invoked as a possible cause of this effect.
In this paper we show, by means of transport and magnetic measurements, low-temperature ferromagnetic quenching effects in $\mathrm{Fe}(110)$ contacting nanometric islands. These effects may be due to either a random spin freezing at the island boundaries or to a weak antiferromagnetic coupling between grains that fluctuations are unable to overcome at low temperatures. As a result, an anomalous increase of the resistivity as well as a decrease of zero-field-cooled (ZFC) $\left(M_{\mathrm{ZFC}}\right)$ magnetization are observed. Ferromagnetic exchange between contacting nanocrystals depends on the capability of the interface to transmit exchange. Therefore, it is inferred that grain boundaries of Fe that transmit ferromagnetic exchange at high temperatures become ferromagnetic exchange insulators at low temperatures.

Granular ferromagnetic thin films formed of nanometersized islands in physical contact are very adequate systems to study both temperature-dependent magnetic interactions as well as transport properties, since a careful control of the growth conditions allows a fine design of the nanostructure. In the present work, $\mathrm{Fe}(110)$ islands were deposited on $\mathrm{Al}_{2} \mathrm{O}_{3}(0001)$ substrates in an ultrahigh-vacuum triode sputtering system at $700{ }^{\circ} \mathrm{C},{ }^{15}$ and a $2.5-\mathrm{nm}$ Pt capping layer was grown at room temperature to protect from oxidation. It should be noted here that in the case of ordered Fe-Pt alloys, which are usually annealed at high temperature (450$\left.500{ }^{\circ} \mathrm{C}\right)$, Pt can give rise to perpendicular anisotropy. ${ }^{16,17}$ However, we did not observe the presence of perpendicular anisotropy in our samples due to the low temperature of the Pt growth necessary to avoid alloying and ordering at the interface Fe-Pt. Island sizes were easily varied simply by changing the deposition time. Three in-plane equivalent azimuthal orientations, ${ }^{1-10} \mathrm{Fe} \|(11-20) \mathrm{Al}_{2} \mathrm{O}_{3}$, as expected from the symmetry of the substrate, are concluded from TEM characterization, ${ }^{15}$ while the out-of-plane orientation for all the islands is (110). Therefore, three equivalent in-plane easy axes separated by $60^{\circ}$ are also expected. If we assume a bulk structure, the easy axis would be $\mathrm{Fe}[001]$; however due to surface anisotropy and/or strain, often present in thin films, the easy axis might be different, although always keeping a 


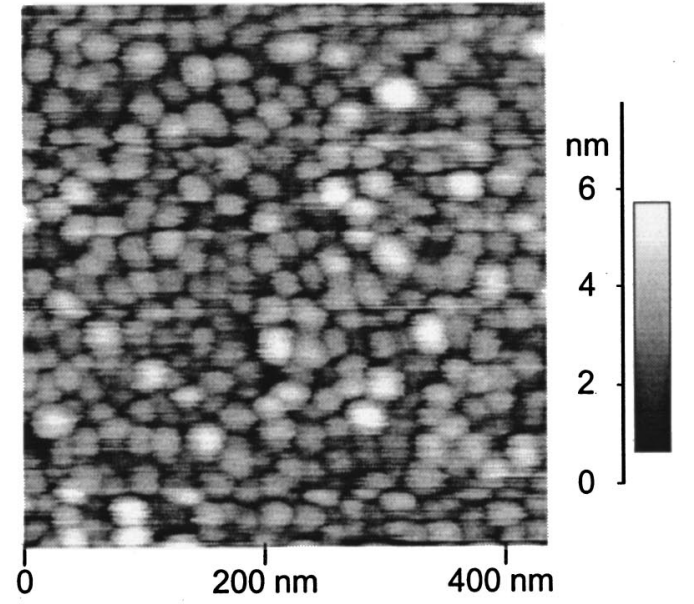

(a)

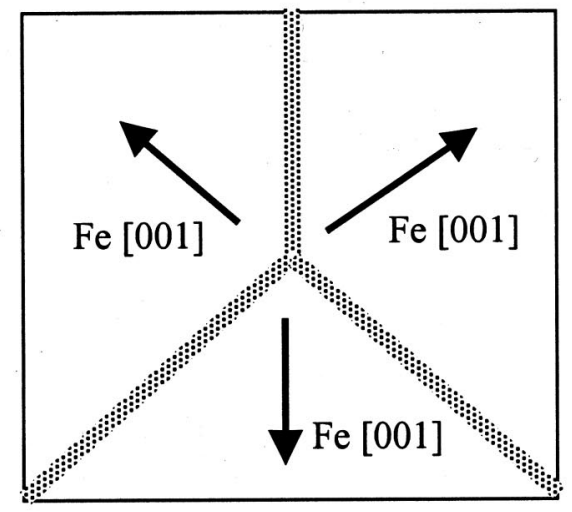

(b)

FIG. 1. (a) AFM image of a selected Fe(110) islanded sample with a 20-nm typical in plane island size. (b) Sketch of the islanded structure showing three in-contact islands with the three equivalent in-plane Fe crystallographic orientations. The shaded area corresponds to the islands boundary regions.

sixfold symmetry due to the crystalline symmetry. In order to test this assertion, magneto-optical Kerr effect loops were performed along different in-plane directions, showing no essential differences in the hysteresis loops, consistent with a highly symmetric sixfold structure. This therefore indicates the presence of a low magnetic anisotropy in this system. Due to the reduced island height for all the fabricated structures (limited to less than $4 \mathrm{~nm}$ ) and the elevated deposition temperature, it is very reasonable to assume that every individual island is a single crystal. An atomic force microscopy (AFM) image for a sample with 20-nm in-plane island size is shown in Fig. 1 (a). The system under study in this paper consists of individual single-crystalline $\mathrm{Fe}(110)$ islands of nanometric dimensions with a discrete in-plane magnetocrystalline anisotropy distribution. In order to confirm that islands are magnetically connected through their bases by an Fe boundary region, Kerr hysteresis loops at room temperature were performed, showing a square shape. This indicates the absence of superparamagnetism, despite the small size of the islands (the maximum volume for the onset of superparamagnetism in bcc $\mathrm{Fe}$ at room temperature is around $\left.10^{3} \mathrm{~nm}^{3}\right)$. The sharpness of the loops is inconsistent with a system being a mixture of superparamagnetic and ferromagnetic regions. Superparamagnetic regions acting independently should give rather smooth loops due to the distribution of local anisotropies, which would result in a distribution of coercive fields. This observation serves as a confirmation that islands observed in the AFM image must be in physical contact, leading to an exchange interaction through the in-contact islands boundaries. In Fig. 1(b) we show a sketch of the fabricated structure illustrating this picture.

The temperature dependence of the electrical resistivity for this system was measured for samples with different island sizes, under applied magnetic fields in the range 0-0.5 $\mathrm{T}$, using a dc four-terminal method. While $\rho(T)$ presents a typical metallic behavior in the whole temperature range for samples with in-plane island diameters $d$, greater than $30 \mathrm{~nm}$, we observe a nonmetallic behavior below a specific temperature $\left(T_{f}\right)$ ranging from 50 to $150 \mathrm{~K}$ for samples with grain sizes varying from $d=26-16.5 \mathrm{~nm}$, respectively, giving rise to a minimum in the resistivity around $T_{f}$. Figure 2(a) shows the temperature dependence of the electrical resistivity with and without magnetic field for a sample with the smallest grain size. It is clear that the upturn of the resistivity disappears under the action of a magnetic field of $0.5 \mathrm{~T}$. Therefore, below $150 \mathrm{~K}$ the system exhibits a decrease of resistivity with further cooling down, up to a value of $5.5 \%$ at $10 \mathrm{~K}$. The results of measurements performed with the same applied magnetic field for a sample with a 26-nm grain size indicate a smaller $(\sim 0.4 \%)$ magnetoresistance. Since such an anomaly completely disappears under the action of a magnetic field, it is inferred that the low-temperature electrical transport is dominated by spin-dependent scattering. In order to confirm the magnetic origin of this phenomenon, we have performed magnetic measurements using a superconducting quantum interference device magnetometer. Figure 2(b) displays the temperature dependence of zero-field-cooled $\left(M_{\mathrm{ZFC}}\right)$ and field-cooled $\left(M_{\mathrm{FC}}\right)$ magnetization under a magnetic field of $0.1 \mathrm{~T}$ for a sample with a $26-\mathrm{nm}$ in-plane island size. While $M_{\mathrm{FC}}$ increases below $50 \mathrm{~K}$, we observe a decrease of $M_{\mathrm{ZFC}}$ below $50 \mathrm{~K}$. A similar dependence of zerofield cooling was observed for a sample with a $16.5-\mathrm{nm}$ inplane island size [see the inset of Fig. 2(b)]. The observation of an upturn in the electrical resistivity, together with a suppression of the magnetization, allow us to conclude that below $T_{f}$ the magnetization becomes inhomogeneous with decreasing temperature. Moreover, the combination of magnetic and transport measurements indicates that the soft ferromagnetic system evolves toward a noncollinear magnetic structure, as the temperature drops below $T_{f}$. This observation also shows the importance of the intergrain exchange in the overall magnetic behavior. For temperatures above $T_{f}$ the spins of the boundary are magnetized along the direction of the adjacent grain by contact to with their molecular field. Thus, the magnetization being homogeneous in the intergrain regions, the whole system is ferromagnetic. Below $T_{f}$ the boundary spins start to freeze toward the ground state reducing the ferromagnetic exchange between islands. This gives rise to a progressive increase of the electrical resistivity due to the enhanced magnetic scattering of 

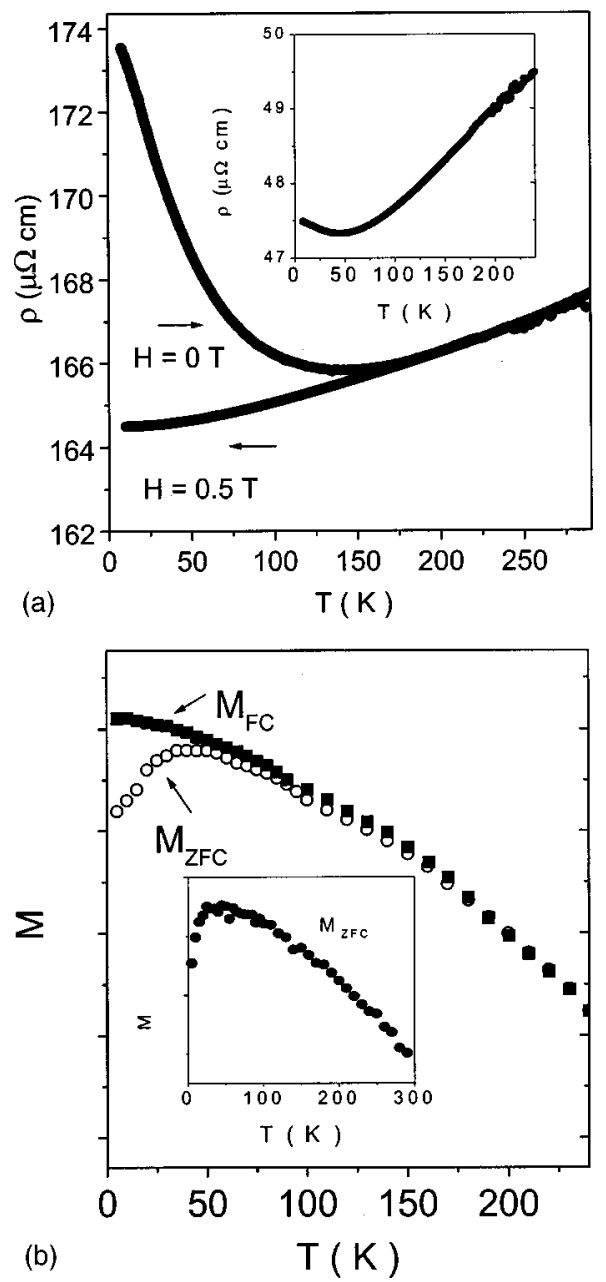

FIG. 2. (a) Temperature dependence of the electrical resistivity at zero magnetic field and in a magnetic field of $0.5 \mathrm{~T}$ for a sample with the smallest obtained grain size $(16.5 \mathrm{~nm})$. Inset: resistance curve at zero magnetic field for an islanded $\mathrm{Fe}(110)$ thin film with an island size of $26 \mathrm{~nm}$. (b) Magnetization $(M)$ vs temperature, measured after zero-field cooling (ZFC) and field cooling (FC) in a magnetic field of $0.1 \mathrm{~T}$ for a sample with an island size of $26 \mathrm{~nm}$. (b) Temperature dependence of the zero-field-cooled $\left(M_{\mathrm{ZFC}}\right)$ magnetization $(0.1 \mathrm{~T})$ for a sample with an island size of $16.5 \mathrm{~nm}$.

the carriers at the grain boundaries. As a consequence of the local anisotropy a random distribution at grains and boundaries appears parallel to the spin freezing, and the total magnetization drops. Further support for a magnetic hardening due to a boundary spin freezing in our nanocrystalline $\mathrm{Fe}$ thin films is based on an analysis of the low-temperature hysteresis loops. In Fig. 3 we show the hysteresis loop at 40 $\mathrm{K}$ for a sample with a $16.5-\mathrm{nm}$ in-plane island size. First of all, let us note that the hysteresis loop is squared at high temperatures, and that the magnetization saturates abruptly at a low magnetic field, indicating a uniform reversal of the magnetization. At low temperature $(40 \mathrm{~K})$, however, the loop is smooth (see Fig. 3), indicating that the boundary gradually reverses its magnetization, and that the boundary spins rotation is not coherent as at high temperature. This is indicated by the fact that the coercive field at $40 \mathrm{~K}$ is one order of magnitude higher than at high temperature. Moreover, the

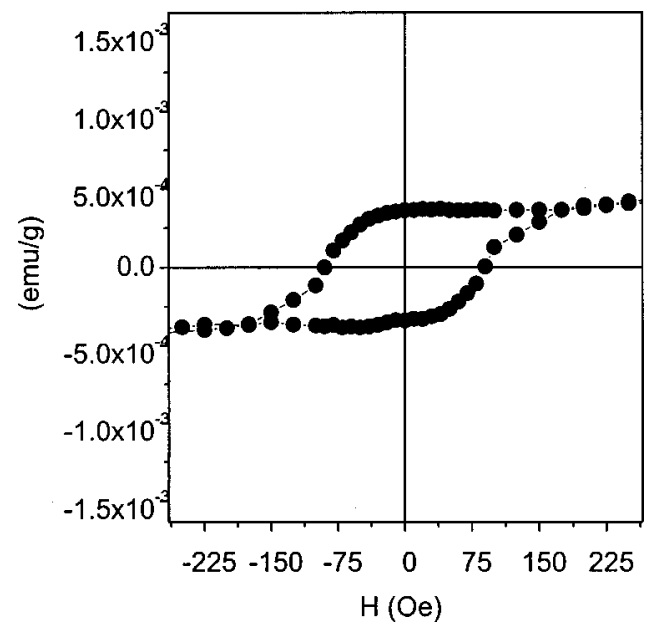

FIG. 3. Hysteresis loop at $40 \mathrm{~K}$ for a samples with $16.5-\mathrm{nm}$ in-plane island sizes.

saturation field, $H_{s}$, increases at low temperature $\left[H_{s}(200 \mathrm{~K}) \approx 30 \mathrm{Oe}\right.$ and $\left.H_{s}(40 \mathrm{~K}) \approx 175 \mathrm{Oe}\right]$ reducing the squareness of the loop, since this quantity is mainly associated with the progressive alignment of the boundary spins toward the field direction. As a result of the above considerations, our data strongly indicate that this soft ferromagnetic system evolves toward a magnetic structure not completely collinear at low temperature.

The key argument about spin freezing was obtained from a generalization of the random-anisotropy model (RAM), ${ }^{13,18}$ for which the self-consistent energy includes reduced intergrain exchange. The basic idea is the competition between three energy scales: (a) the anisotropy energy within a finite volume island, (b) the exchange energy for adjusting the magnetization between such island, and (c) the exchange energy across grain boundaries. According to a two-phase generalized RAM, ${ }^{13,18}$ the ferromagnetic exchange between two adjacent islands is given by $\gamma A$, where $A$ is the exchange coupling coefficient for bcc Fe, typically $A=10^{-11} \mathrm{~J} \mathrm{~m}^{-1}$, and $\gamma$ is a factor, ranging between 0 and 1 , which accounts for the possible modification of the interactions introduced by the interface structure. The exchange correlation length through the intergrain boundary, $L^{*}$ is of the order of $(\gamma A / k)^{1 / 2}$, where $k$ is the experimental anisotropy constant of bcc Fe, $k=4.8 \cdot 10^{4} \mathrm{~J} \mathrm{~m}^{-3}$ at room temperature. ${ }^{19}$ If $L^{*}$ exceeds the maximum grain dimension $d$, the magnetization does not follow the easy direction of each individual grain, but tends to align itself along a common easy axis. Note that $L^{*}>d$ implies that $\gamma>d^{2} K / A$, and therefore there exists a ferromagnetic exchange between islands, $\gamma \sim 1$, and the whole system is ferromagnetic and soft. Since experimentally we observe that $L^{*}$ decreases with cooling, there could be a temperature for which $L^{*}$ scales $d$, thus giving rise to a progressive inhomogeneous magnetization for further decreasing temperature. According to this argument, the lowtemperature progressive inhomogeneous magnetization of the phases must be due to a decrease of $\gamma$.

In summary, we have investigated a magnetic thin-film 
granular system that exhibits magnetic and transport properties that strongly depend on the intergrain exchange. The decrease of magnetization at low temperatures, similar to that previously observed in nanopowders of ball-milled Fe, indicates that it is a characteristic of nanocrystalline $\mathrm{Fe}$, independent of the production method. On the other hand, the existence of a minimum for resistivity due to a random arrangement of the individual magnetization has been ob

*Corresponding author: Z. Sefrioui. Email address: sefrioui@imm.cnm.csic.es

${ }^{1}$ M. N. Baibich, J. M. Broto, A. Fert, F. Nguyen Van Dau, F. Petroff, P. Etienne, G. Creuzet, A. Friederich, and J. Chazelas, Phys. Rev. Lett. 61, 2472 (1988).

${ }^{2}$ P. Levy, S. Zhang, and A. Fert, Phys. Rev. Lett. 65, 1643 (1990).

${ }^{3}$ S. S. P. Parkin, N. More, and K. P. Roche, Phys. Rev. Lett. 64, 2304 (1990).

${ }^{4}$ G. Binasch, P. Grünberg, F. Saurenbach, and W. Zinn, Phys. Rev. B 39, 4828 (1989).

${ }^{5}$ J. S. Moodera, L. R. Kinder, T. M. Wong, and R. Meservey, Phys. Rev. Lett. 74, 3273 (1995).

${ }^{6}$ B. Dieny, V. S. Speriosu, S. S. P. Parkin, B. A. Gurney, D. R. Wilhoit, and D. Mauri, Phys. Rev. B 43, 1297 (1991).

${ }^{7}$ A. E. Berkowitz, J. R. Mitchell, M. J. Carey, A. P. Young, S. Zhang, F. E. Spada, F. T. Parker, A. Hutten, and G. Thomas, Phys. Rev. Lett. 68, 3745 (1992).

${ }^{8}$ J. Q. Xiao, J. S. Jiang, and C. L. Chien, Phys. Rev. Lett. 68, 3749 (1992).

${ }^{9}$ A. Milner, A. Gerber, B. Groisman, M. Karpovsky, and A. Gladkikh, Phys. Rev. Lett. 76, 475 (1996). served. The amplitude of the above effects and $T_{f}$ itself strongly depend on the grain size in the region of $16-26 \mathrm{~nm}$.

\section{ACKNOWLEDGMENTS}

Z.S. and J.L.M. acknowledge the Comunidad de Madrid for financial support. Work was performed under the financial support of the Comunidad de Madrid and the Spanish Commission of Science and Technology.

${ }^{10}$ H. Y. Hwang, S.-W. Cheong, N. P. Ong, and B. Batlogg, Phys. Rev. Lett. 77, 2041 (1996).

${ }^{11}$ J. M. D. Coey, A. E. Berkowitz, L1. Balcells, F. F. Putris, and A. Barry, Phys. Rev. Lett. 80, 3815 (1998).

${ }^{12}$ J. F. Gregg, W. Allen, K. Ounadejla, M. Viret, M. Hehn, S. M. Thompson, and J. M. D. Coey, Phys. Rev. Lett. 77, 1580 (1996).

${ }^{13}$ J. F. Löffler, H. B. Braun, and W. Wagner, Phys. Rev. Lett. 85, 1990 (2000).

${ }^{14}$ E. Bonetti, L. Del Bianco, D. Fiorani, D. Rinaldi, R. Caciuffo, and A. Hernando, Phys. Rev. Lett. 83, 2829 (1999).

${ }^{15}$ J. L. Menéndez, G. Armelles, C. Quintana, and A. Cebollada, Surf. Sci. (to be published).

${ }^{16}$ B. M. Lairson, M. R. Visokay, R. Sinclair, and B. M. Clemens, Appl. Phys. Lett. 62, 639 (1993).

${ }^{17}$ A. Cebollada, D. Weller, J. Sticht, G. R. Harp, R. F. C. Farrow, R. F. Marks, R. Savoy, and J. C. Scott, Phys. Rev. B 50, 3419 (1994).

${ }^{18}$ A. Hernando, M. Vázquez, T. Kulik, and C. Prados, Phys. Rev. B 51, 3581 (1995).

${ }^{19}$ B. D. Cullity, Introduction to Magnetic Materials (AddisonWesley, Reading, MA, 1972). 